14. Bahmanimehr A, Nikmanesh F, Yepiskoposyan L. Paternal genetic landscape of Zagros region of Iran and its role in the gene flow in the populations of the region. Iran J Epidemiol 2014;10(3):43-53.

15. Vigne J-D. The origins of animal domestication and husbandry: a major change in the history of humanity and the biosphere. Comptes rendus biologies 2011;334:171-181.

16. Esmaeili H. Brucellosis in the Islamic republic of Iran. J Med Bacteriol 2014;3(3-4):47-57.
17. Honarvar B, Moghadami M, Lankarani K, et al. Brucellosis as a neglected disease in a neglected population: a seroepidemiological study of migratory nomads in the Fars province of Iran. Epidemiol Infect 2017;145:491-497.

18. Franc K, Krecek R, Häsler B, Arenas-Gamboa A. Brucellosis remains a neglected disease in the developing world: a call for interdisciplinary action. BMC Pub Health 2018;18:125.

19. Agriculture. Statistical Center of Iran website. https://www.amar.org.ir/ english/Statistics-by-Topic/Agriculture\#113281-releases. Published 2019. Accessed December 30, 2019.

\title{
Sustaining outpatient antimicrobial stewardship: Do we need to think further outside the box?
}

\author{
Holly M. Frost MD ${ }^{1,2}$ (D), Leisha M. Andersen MD, MPH ${ }^{3}$, Katherine E. Fleming-Dutra MD², Chuck Norlin $\mathrm{MD}^{5}$ and \\ Christopher A. Czaja MD, MPH ${ }^{6}$ \\ ${ }^{1}$ Department of Pediatrics, Denver Health Medical Center, Denver, Colorado, ${ }^{2}$ Department of Pediatrics, University of Colorado School of Medicine, Aurora, \\ Colorado, ${ }^{3}$ American Academy of Pediatrics- Colorado Chapter, Denver, Colorado, ${ }^{4}$ Division of Healthcare Quality Promotion, Centers for Disease Control and \\ Prevention, Atlanta, Georgia, ${ }^{5}$ Department of Pediatrics, University of Utah, Salt Lake City, Utah and ${ }^{6}$ Colorado Department of Public Health and Environment, \\ Denver, Colorado
}

To the Editor-Antibiotic overuse is a persistent public health problem that has contributed to an alarming increase in the prevalence of antibiotic resistance. ${ }^{1}$ In the United States, $>70 \%$ of antibiotic courses are prescribed in ambulatory settings. ${ }^{2}$ To encourage the appropriate use of antibiotics in outpatient practices, the Centers for Disease Control and Prevention (CDC) released the Core Elements of Outpatient Antibiotic Stewardship (hereafter Core Elements). ${ }^{3}$ However, detailed evaluations of how practices implement and sustain the Core Elements are lacking.

From January through June 2019, an antimicrobial stewardship quality improvement initiative was conducted by the American Academy of Pediatrics Chapter Quality Network (AAP-CQN), Colorado Department of Public Health and Environment, and the CDC. In this project, 8 outpatient pediatric practices in Colorado were enrolled: 3 practices that were part of larger systems and 5 independent practices. All practices but 1 were urban or suburban. The initiative utilized the Core Elements and Model for Improvement frameworks. ${ }^{3}$ Practices implemented interventions to improve prescribing for pharyngitis and acute otitis media. The AAP-CQN provided webinars, learning sessions, monthly tracking and reporting, and Maintenance of Certification (MOC) credit. The ability of the practices to continue antibiotic stewardship following the conclusion of the initiative was assessed.

Using surveys and telephone-based semistructured interviews with physician champions, we assessed the ability of practices to sustain the Core Elements of commitment, tracking and reporting, and education and expertise. Because the initial intervention focused on evaluation of the Core Element "action for policy and practice," our interviews targeted the other Core Elements. The project was determined exempt by the AAP Institutional Review Board.

Author for correspondence: Holly M. Frost, E-mail: Holly.frost@dhha.org

Cite this article: Frost HM, et al. (2020). Sustaining outpatient antimicrobial stewardship: Do we need to think further outside the box?. Infection Control \& Hospital Epidemiology, 41: 382-384, https://doi.org/10.1017/ice.2019.366
Most practices identified a leader for antibiotic stewardship (7 of 8) and intended to display commitment posters (5 of 7). ${ }^{4}$ Most had access to antibiotic expertise (6 of 8) and planned to provide ongoing education regarding antibiotic use (6 of 8). Few practices had established systems for ongoing antibiotic stewardship-related duties (4 of 8), tracking (5 of 8), or reporting (3 of 7).

Interviews identified 6 barriers to sustaining the 3 Core Elements (Table 1). Lack of control and competing priorities complicated practices' capacity to display commitment. Of the 5 practices reporting implementation of a commitment poster on the survey, only 2 affirmed the finding during interviews. Barriers to displaying posters included modifying the CDC template, inserting provider photos, and printing logistics. For practices that were part of a larger healthcare system, the need to obtain administrative approval (for information technology (IT) support or to hang commitment posters) was a key limitation.

Barriers to tracking and reporting included time constraints and lack of information technology (IT) support. Only 2 practices had IT support. Practices reported that manual or electronic abstraction took substantial time (range, $4-20$ hours by a physician or clinic manager). Consequently, 5 practices did not plan to continue tracking and reporting, and 3 practices planned abbreviated and less frequent (every 3-6 months) tracking and reporting. Practices were most likely to sustain tracking and reporting if a nonphysician was responsible for data.

Barriers to sustaining education/expertise included scheduling and lack of resources (eg, regional experts). In the absence of MOC and outside support, the structures of most practices did not support scheduled education on antibiotic stewardship.

To sustain antibiotic stewardship, practices would benefit from increased availability of practical strategies and tools. Strategies to support the commitment to the Core Elements might include a centralized online system for practices to design and order custom commitment posters. For our initiative, AAP-Colorado created and supplied posters, which were well received. Because tracking and 
Table 1. Qualitative Survey Results Assessing Sustainability of Antibiotic Stewardship Efforts From 8 Colorado Practices Participating in an Intensive American Academy of Pediatrics Chapter Quality Network (AAP CQN) and Centers for Disease Control and Prevention (CDC) Antibiotic Stewardship Intervention, July 2019

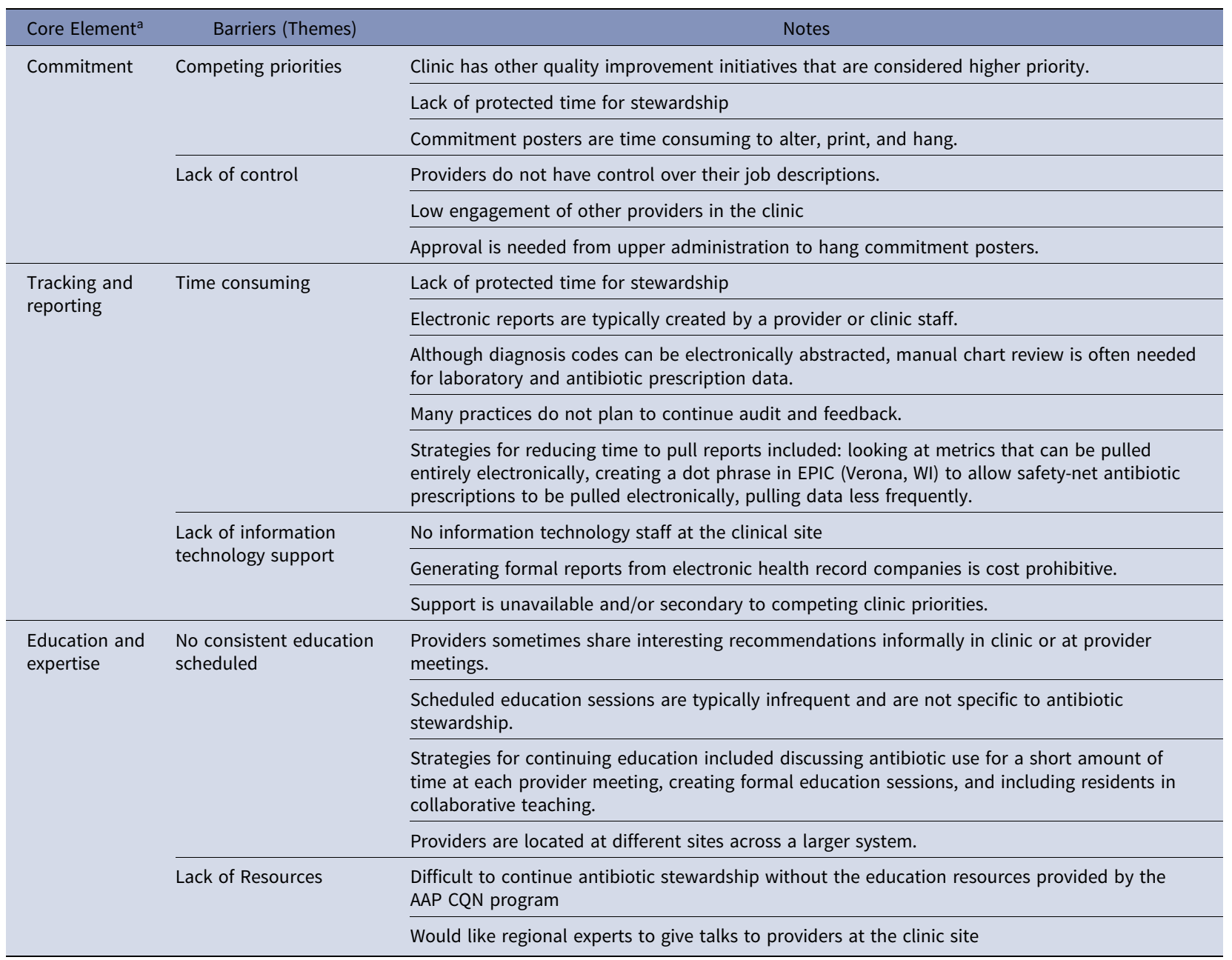

action for policy and practice were implemented as part of the AAP CQN collaborative work and were not included in the sustainability assessment of the Core Elements.

reporting (with individualized provider feedback) is the most effective way to reduce antibiotic use, ${ }^{5,6}$ practices need support to efficiently use metrics for electronic data tracking. Strategies to improve tracking and reporting might include reducing the frequency of tracking and reporting, using a point-prevalence survey, ${ }^{7}$ creating tip sheets for electronic health records, and promoting an online forum focused on efficiently pulling data. In this initiative, practices benefitted from sharing tips on how to pull data. To facilitate ongoing stewardship-focused education and expertise, practices could incorporate brief opportunities for stewardship-based education into their routine schedule. Alternative approaches might include virtual learning communities or use of MOC. Practices in our initiative found success in routinely setting aside 5-10 minutes at provider meetings to discuss antibiotic use or asking medical students or residents to present relevant topics.

This study has several strengths including coordinated national expertise in antimicrobial stewardship that included the involvement of the AAP, state health department, CDC, and highly engaged practices. The study was limited by a small sample, which may have reduced generalizability of the results. However, the challenges identified here likely reflect those at other community- based outpatient clinics. Future steps will include evaluating antibiotic stewardship practices 6 months after the initiative.

In conclusion, although the Core Elements provide a framework for outpatient antibiotic use, further work is needed to develop practical strategies and tools that are simple, evidence based, high impact, and sustainable. In this project, implementing even seemingly simple interventions, such as adding photos to a commitment poster, posed logistical challenges that became barriers. Although several publications ${ }^{3,8}$ offer various suggestions to improve outpatient stewardship efforts, ambulatory practices would benefit from additional clear, step-by-step guidance and enhanced sustainability tool kits, analogous to the playbook for antibiotic stewardship produced for hospitals. ${ }^{9}$ We consider a few potential approaches to sustaining outpatient stewardship here, but a broader discussion among antibiotic stewards, patient care organizations, public health, and other stakeholders will be important for future success.

Acknowledgments. We are grateful for the tremendous support provided by Ellen Brilliant, Jennifer Monti, and Suzanne Emmer from the American Academy of Pediatrics throughout this project. The findings and conclusions in this manuscript are those of the authors and do not necessarily represent 
the official position of the Centers for Disease Control and Prevention Colorado Department of Public Health and Environment, or the American Academy of Pediatrics.

Financial Support. This project was supported by the Centers for Disease Control and Prevention (CDC) through a Cooperative Agreement (grant no. 6NU38OT000292-01-01) with the Chapter Quality Network, a program of the American Academy of Pediatrics. H.F. received salary support from the National Institutes of Health (NICHD grant no. 1K23HD099925-01A1).

Conflicts of Interest. The authors have no conflicts of interest to disclose.

\section{References}

1. Antibiotic/Antimicrobial resistance. Centers for Disease Control and Prevention website. http://www.cdc.gov/drugresistance/index.html. Published 2016. Accessed July 11, 2016.

2. Suda KJ, Hicks LA, Roberts RM, Hunkler RJ, Matusiak LM, Schumock GT. Antibiotic expenditures by medication, class, and healthcare setting in the United States, 2010-2015. Clin Infect Dis 2018;66:185-190.

3. Sanchez GV, Fleming-Dutra KE, Roberts RM, Hicks LA. Core elements of outpatient antibiotic stewardship. Morbid Mortal Wkly Rept 2016;65:1-12.
4. Meeker D, Knight TK, Friedberg MW, et al. Nudging guideline-concordant antibiotic prescribing: a randomized clinical trial. JAMA Intern Med 2014;174:425-431.

5. Gerber JS, Prasad PA, Fiks AG, et al. Durability of benefits of an outpatient antimicrobial stewardship intervention after discontinuation of audit and feedback. JAMA 2014;312:2569-2570.

6. Meeker D, Linder JA, Fox CR, et al. Effect of behavioral interventions on inappropriate antibiotic prescribing among primary care practices: a randomized clinical trial. JAMA 2016;315:562-570.

7. Frost HM, Knepper BC, Shihadeh KC, Jenkins TC. A novel approach to evaluate antibiotic utilization across the spectrum of inpatient and ambulatory care and implications for prioritization of antibiotic stewardship efforts. Clin Infect Dis 2019. doi: 10.1093/cid/ciz466.

8. Improving antibiotic prescribing for children, change package. American Academy of Pediatrics CQN website. https://downloads.aap.org/DOCCSA/ CQN\%20ABX\%20Change\%20Package\%20Final\%20October\%202019.pdf. Published 2019. Accessed November 1, 2019.

9. National Quality Partners playbook: antibiotic stewardship in acute care. National Quality Forum website. https://store.qualityforum.org/collections/ antibiotic-stewardship/products/national-quality-partners-playbook-antibioticstewardship-in-acute-care. Published 2016. Accessed November 1, 2019. 